\title{
Use of ambulance dispatch calls for surveillance of severe acute respiratory infections?
}

\author{
Susana Monge ${ }^{1,2}$, Janneke Duijster ${ }^{1}$, Geert Jan Kommer ${ }^{1}$, Jan van de Kassteele ${ }^{1}$, Gé \\ Donker $^{3}$, Thomas Krafft ${ }^{4}$, Paul Engelen ${ }^{5}$, Jens P. Valk ${ }^{6,7}$, Jan de Waard ${ }^{8}$, Jan de Nooij ${ }^{8}$, Wim \\ van der Hoek ${ }^{1}$, Liselotte van Asten ${ }^{1}$ \\ ${ }^{1}$ Centre for Infectious Disease Control Netherlands, National Institute for Public Health and the Environment (RIVM), Utrecht, Bilthoven, \\ Netherlands, ${ }^{2}$ European Centre for Disease Prevention and Control, (ECDC), Stockholm, Sweden, ${ }^{3}$ Nivel Primary Care Database - sentinel \\ practices, Utrecht, Netherlands, ${ }^{4}$ Maastricht Centre for Global Health, Maastricht University, Maastricht, Netherlands, ${ }^{5}$ Meldkamersupport, \\ Hellevoetsluis, Netherlands, ${ }^{6}$ Dispatch Center Regional Ambulance Services Noord Nederland, Drachten, Netherlands, ${ }^{7}$ University Medical \\ Center Groningen, Groningen, Netherlands, ${ }^{8}$ Regional Ambulance Service Hollands Midden, Den Haag, Netherlands
}

\section{Objective}

We aim to assess whether influenza circulation, as measured through influenza-like-illness (ILI) in primary care, is reflected in ambulance dispatch (AD) calls.

\section{Introduction}

Surveillance of severe influenza infections is lacking in the Netherlands. Ambulance dispatch (AD) data may provide information about severity of the influenza epidemic and its burden on emergency services. The current gold standard, primary care-based surveillance of influenza-like-illness (ILI), mainly captures mild to moderate influenza cases, and does not provide adequate information on severe disease. Monitoring the severity of the annual epidemic, particularly among groups most at risk of complications, is of importance for the planning of health services and the public health response.

\section{Methods}

We analysed all calls from four ambulance dispatch centers serving 4.3 million people in the Netherlands, between January 2014 and December 2016. The main complaint and urgency level is recorded during triage; those possibly caused by respiratory infections were grouped as respiratory syndrome calls (RSC). We modelled the proportion of all RSC calls against the weekly ILI incidence (we allowed up to 4-week lags and leads), from sentinel primary-care surveillance. We used binomial regression with identity link to obtain differences in proportions. We built separate models by age group, urgency level and time of day. We tested heterogeneity of effects by season.

\section{Results}

We included 289,307 calls; $6.7 \%$ were RSC. Overall, proportion of RSC increased by 0.114 percentage points for each increase of 1/10,000 population in ILI incidence. In our study population, this translated into 550 ambulance calls attributable to influenza (as measured by ILI) per year. Association was stronger in the models including only out-of-office hours, children ( $<15$ years) and highest urgency level calls. In the latter two, the effect varied by season. RSC was best associated with ILI from the previous 1-3 weeks in all models, except in children where RSC preceded ILI by 1 week.

\section{Conclusions}

Our results demonstrate the potential usefulness of ambulance dispatch data to complement existing influenza surveillance by providing information on the volume and timing of severe cases attributable to influenza within the yearly epidemics. 